\title{
COMPOSIÇÃO DA COLEOPTEROFAUNA EM AMBIENTES COM DIFERENTES SISTEMAS DE MANEJO, UM ENFOQUE NA ATRATIVIDADE
}

MOREIRA, Edimar Agnaldo ${ }^{1}$ edimarbio@ hotmail.com, PINTO Gabriel Silva² gabriel60pinto@ gmail.com.

1. Pós Graduando em Ecologia e Tecnologia Ambiental, Universidade Federal de Alfenas - UNIFALMG;

2. Professor Doutor docente da Faculdade de Ciências e Tecnologias de Campos Gerais FACICA-MG.

\section{Recebido em: 25/05/2014 - Aprovado em: 30/06/2014 - Disponibilizado em: 30/07/2014}

\begin{abstract}
RESUMO: A Ordem Coleóptera compreende o grupo taxonômico mais diverso entre os insetos em todo mundo, com um número de espécies em torno de 357.000, entretanto o conhecimento sobre eles é pequeno comparado a sua diversidade. Objetivamos com este trabalho pesquisar a fauna de Coleópteros em diferentes ambientes os quais possuíam variados níveis de alteração ambiental. Para a realização do estudo foram escolhidas 3 áreas sendo, um Fragmento de mata, uma monocultura de café e uma segunda monocultura cafeeira, porém, em processo de poda. Foram realizadas 8 coletas, todas em uma única estação do ano, no período de outubro a novembro de 2012. Utilizou-se de 2 métodos de coletas, para besouros de solo: pitfall com diferentes atrativos e, para besouros voadores, Pet Santa Maria iscada com álcool 98\%. Foram amostrados 1174 besouros agrupados em 12 famílias. O Fragmento de mata apresentou a maior diversidade de famílias coletadas, seguido pela monocultura de café com poda. A família Scarabaeidae foi a mais abundante em todas as coletas. Quanto aos atrativos a preferência por fezes humanas foi representativa, pois dos 1014 Coleópteros capturados por pitfall 914 foram atraídos por fezes humanas. À medida que a alteração do ambiente progride a diversidade tende a regredir, com isso podemos ver que a grande diversidade de coleóptera está ameaçada com essas modificações nos ecossistemas.
\end{abstract}

PALAVRAS-CHAVE. Besouros. Atratividade. Riqueza de famílias.

ABSTRACT: Coleoptera comprises the most diverse taxonomic group of insects in the world, with a number of species about 357,000. Nevertheless the knowledge related to them is small compared to its diversity. The scope of the present study is to survey the Coleoptera fauna living in different environments with a varying levels of environmental alteration. A Forest patch, a monoculture of coffee and a second coffee monoculture, however, in a process of pruning. Eight field works were made and all of them did in a single season, from October to November 2012. Using 2collection methods for ground beetles, a pitfall trap with different attractive and for flying beetles, a Pet Santa Maria trap baited with $98 \%$ alcohol. We sampled 1174 beetles grouped into 12 families. The forest patch showed the greatest diversity of families collected, followed by monoculture with pruning. The family Scarabaeidae was the most abundant in all samples. Results for the preference for attractive bait, human feces was more representative, because from 1014 beetles captured by pitfall 914 were attracted by human feces. As environmental changing is increasing the Coleoptera diversity tends to regress and consequently it was possible to observe that the great diversity of Coleoptera is threatening by alterations in ecosystems.

KEYWORDS. Beetles. Attractiveness. Richness of families.

\section{INTRODUÇÃO}

O Brasil apresenta a maior

biodiversidade do planeta, entretanto a intensa utilização de monoculturas aliada ao processo de fragmentação das matas resultados da agricultura são fatores que levamos ecossistemas a perderem sua complexidade refletindo na extinção das espécies (COPATTI \& DAUT, 2009).

$$
\text { Estudos demonstraram que a }
$$

composição da fauna é diretamente influenciada pelas diferentes estruturas da vegetação (DOUBE \& WARDHALGH, 
1991). Isto ocorre pela alteração de características ambientais que agem direta ou indiretamente na biologia de uma população. Por exemplo, a luminosidade, a temperatura, umidade e outras condições ambientais que estão ligadas à reprodução, o forrageamento e ao próprio desenvolvimento dos organismos assim como das comunidades (DOUBE \& WARDHALGH, 1991). Outro fator que influencia na fauna é o uso do solo pelo homem, o que acarreta alterações na composição e diversidade de organismos em diferentes graus de intensidade. Estas modificações no habitat são evidenciadas no fornecimento de alimento, na presença de micro-climas e interações intra e interespecíficas (FREITAS \& BARRETO, 2008).

Devido a grande intensidade de alterações, principalmente perturbações antrópicas aos ecossistemas naturais, a conservação da biodiversidade, vem sendo um grande desafio atualmente. A consequência mais marcante dessas alterações é a fragmentação dos habitats. Tendo a Mata Atlântica como exemplo, a grande maioria dos remanescentes florestais, principalmente paisagens intensamente cultivadas, encontram-se na forma de pequenos fragmentos, intensamente perturbados, isolados, pouco conhecidos e menos ainda protegidos (FREITAS \& BARRETO, 2008 apud NAZARO et al., 2010).

A ação humana como, por exemplo, a intensa mecanização da agricultura, uso de agrotóxicos, pesticidas, etc., e técnicas de colheitas, prejudicam os métodos naturais de dispersão, o controle biológico natural das comunidades de insetos e modificam os níveis de decomposição e fertilidade do solo, atuando assim negativamente sobre a diversidade local (ALTIERI, 1995 apud OLIVEIRA, 2012).

Modificação e fragmentação de habitats são os dois tipos mais comuns de conversão da paisagem, estes processos, envolvem a reconfiguração de um habitat em pequenas e isoladas partes. As áreas abertas criadas com diversos propósitos como a agricultura, por exemplo, nas florestas naturais, originam ambientes fragmentados e condições microclimáticas distintas (maior intensidade luminosa, incidência de ventos e amplitude térmica) devido à falta da cobertura vegetal. Essas modificações microclimáticas causam um declínio na biodiversidade (COSTA et al., 2009).

Já nas florestas, por possuírem grande cobertura vegetal, reduzem a compactação e a erosão do solo através do amortecimento do impacto da chuva com o solo, e as copas das árvores reduzem a velocidade dos ventos 
(CASTRO et al., 1996). Propiciam vários micro-climas favoráveis em termos de umidade, temperatura e incidência solar para a permanência e desenvolvimento de uma maior diversidade de organismos (DAVIS, 2002).

O levantamento de Coleópteros para o monitoramento da qualidade ambiental, fornece subsídios para o desenvolvimento de planos de manejo sustentável. Esse grupo de insetos permite a manutenção do ecossistema em longo prazo e seu monitoramento é econômica e ecologicamente viável. Tendo como incógnita o efeito dessas diferentes formações vegetais e das alterações causadas pelo homem sobre a diversidade de Coleópteros, assim a proposta do trabalho foi analisar os fatores ambientais, alteração ambiental e cobertura vegetal, que podem estar relacionados com a abundância e diversidade de coleóptera na comunidade rural Mato Dentro.

\section{MATERIAL E MÉTODOS}

A área de estudo está localizada na zona rural do município de Campos Gerais, MG conhecida como Comunidade Mato Dentro, está situada na porção sul do estado de Minas Gerais, entre as coordenadas geográficas Latitude Sul 21¹5'11.68" e
Longitude Oeste $45^{\circ} 46 ' 50.89$ e altitude de 876 metros.

Para a realização do estudo foram escolhidas 3 áreas com diferentes tipos de alteração ambiental, um Fragmento Florestal, Latitude Sul $21^{\circ} 15^{\prime} 16.62^{\prime \prime}$ e Longitude Oeste $45^{\circ} 46^{\prime} 47.98^{\prime \prime}$ e altitude de 869 metros. O segundo ponto de coleta é uma área cultivada com a monocultura de café (Coffea arábica), Latitude Sul $21^{\circ} 15^{\prime} 14.29 "$, Longitude Oeste $45^{\circ} 466^{\prime} 52.59 "$ e altitude de 877 metros. O terceiro ponto é também uma área cultivada com Coffea arabica, porém, em estágio de poda. Esta área foi selecionada por ser igual a segunda, porém, alterada pelo processo de poda (trato cultural agrícola denominado esqueletamento).

Foram utilizadas armadilhas de queda (MILHOMEN et al., 2003, FAVILA; HALFFTER, 1997 apud OLIVEIRA MENDONÇA, 2011) e de interceptação de voo, ambas com atrativo, armadilhas de queda basicamente se definem por um recipiente enterrado com a borda nivelada ao nível do solo possuindo em seu interior uma solução (água, sal e detergente) capaz de matar e conservar os indivíduos coletados podendo conter ou não algum atrativo para insetos (AQUINO, 2006).

As pitfalls possuíam dimensões de 15 $\mathrm{cm}$ de diâmetro e $20 \mathrm{~cm}$ de profundidade eum 
telhado composto por uma cobertura de madeira apoiada por palitos a $10 \mathrm{~cm}$ de altura do solo e apresentando certa inclinação, para proteger de sol excessivo, que secaria a isca, ou chuva, que faria a armadilha transbordar.

A armadilha de interceptação de voo, intitulada Pet Santa Maria é composta por uma garrafa pet de dois litros que forma o corpo principal da armadilha, uma parede de interceptação de voo dos insetos confeccionado por plástico transparente 19 $\mathrm{cm}$ x $9 \mathrm{~cm}$, um prato plástico de $21,5 \mathrm{~cm}$ de diâmetro, utilizado como proteção contra chuva e possíveis quedas de galhos folhas e sementes. Uma mangueira, no centro da armadilha, formando um arco, contendo 6 mililitros de álcool 98\% GL, o qual é utilizado como atrativo e liberado pela evaporação nas extremidades da mangueira. $\mathrm{O}$ recipiente de coleta de insetos foi composto por uma garrafa pet de $600 \mathrm{ml}$, na qual é acrescentado $250 \mathrm{ml}$ de álcool $70 \%$ para a conservação dos insetos capturados

Ao todo 27 armadilhas de queda foram utilizadas no estudo, as quais, foram distribuídas nos 3 campos de coleta, as áreas 1, 2 e 3 receberam cada, 9 armadilhas de queda (pitfall) com atrativo, distribuídas em um conjunto, espaçadas $10 \mathrm{~m}$ entre si. Cada conjunto com nove pitfalls recebeu 4 iscas de fezes bovinas, 4 iscas de fezes humanas e 1 sem qualquer tipo de atrativo para fins de controle e 1 armadilha de interceptação de vôo no centro de cada área de amostragem.

Nas monoculturas as pitfalls foras distribuídas em 3 transectos espaçados a 10m, cada transecto continha 3 armadilhas com atrativos alternados, de modo que a pitfall controle localizava-se sempre ao centro. No fragmento florestal foi utilizado um único transecto portando as 9 armadilhas de queda espaçadas a 10 metros e com atrativos alternados, permanecendo com a pitfall controle no cento do local. As armadilhas tipo Pet Santa Maria foram instaladas no interior e no centro de cada área de coleta.

As coletas, bem como a reposição de iscas e solução conservante foram realizadas a cada 48 horas todas em uma única estação do ano no caso a Primavera Outubro/Novembro de 2012. A identificação dos espécimes foi realizada até o nível de família seguindo a chave de identificação de Carrera 1976. O material coletado encontra-se depositado no Laboratório de Zoologia da Faculdade de Ciências e Tecnologias de Campos Gerais.

\section{RESULTADOS E DISCUSSÃO}

Após os trabalhos de campo foram coletados 1.174 Coleópteros classificados em 12 famílias. Constatamos que o esforço 
amostral foi satisfatório, pois a curva do gráfico de acumulação mostrou tendência à estabilização a partir da quarta e quinta coletas (Figura 1). A família Scarabaeidae apresentou $86,37 \%$ dos indivíduos coletados nos 3 pontos de coleta, seguida pelas famílias Cerambycidae $(3,66 \%)$, Lampyridae $(2,47 \%)$, Curculionidae $(2,21 \%)$, Bostrichidae com $2,12 \%$ as outras famílias se mantiveram com menos de 1\% (Figura 2).

Figura 1. Curva de acumulação, utilizando a ordem de observação dos dados com a média das curvas dos 3 pontos de coleta na Comunidade rural Mato Dentro, Campos Gerais, MG.

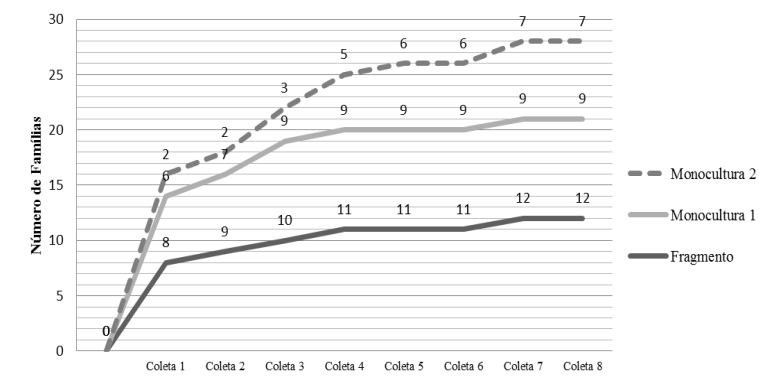

Fonte - Moreira (2013).

Quanto a abundância da coleopterofauna, a família Scarabaeidae foi a que apresentou maior representatividade, totalizando 1014 indivíduos o que corresponde a $86,37 \%$ de todas as famílias amostradas. De acordo com Bianchim et al. (1998), os escarabeídeos apresentam importante papel ecológico na manutenção dos ecossistemas, por utilizarem matéria orgânica em decomposição para alimentação de larvas e adultos, principalmente excrementos (coprofagia) e carcaças (necrofagia), com isso contribuem para processos de ciclagem de nutrientes, bioturbação do solo, destruição de habitas de parasitas, dispersão secundária de sementes entre outras funções ( NICHOLS et al., 2008 apud AUDINO et al 2011).

Figura 2. Distribuição e especificação das famílias amostradas nos 3 pontos de coleta na Comunidade rural Mato Dentro, Campos Gerais, MG. (A.Q, armadilha de queda; A.I.V., armadilha de interceptação de voo; F.H., fezes humanas; F.B., fezes bovinas e P.C. pitfall controle).

\begin{tabular}{|c|c|c|c|c|c|c|c|c|c|c|c|c|c|c|c|}
\hline \multirow{3}{*}{ FAḾ́LIAS } & \multicolumn{5}{|c|}{ FRAGMENTO } & \multicolumn{5}{|c|}{ MONOCULTURA 1} & \multicolumn{5}{|c|}{ MONOCULTURA 2} \\
\hline & \multicolumn{3}{|l|}{ A.Q. } & \multirow{2}{*}{ A.I.v. } & \multirow{2}{*}{ Total } & \multicolumn{3}{|l|}{ A.Q } & \multirow{2}{*}{ A.I.V. } & \multirow{2}{*}{ Total } & \multicolumn{3}{|l|}{ A.Q } & \multirow{2}{*}{ A.I.V. } & \multirow{2}{*}{ Total } \\
\hline & F.H & F.B & P.C. & & & F.H & F.B & P.C. & & & F.H & F.B & P.C. & & \\
\hline Scarabaeidae & 252 & 2 & & 2 & 256 & 392 & 16 & 5 & & 413 & 270 & 70 & 3 & 2 & 345 \\
\hline Cerambycidae & 3 & & & 8 & 11 & & & & 16 & 16 & & & & 16 & 16 \\
\hline Lampyridae & 22 & 3 & & & 25 & 1 & & & & 1 & 1 & 2 & & & 3 \\
\hline Curculionidae & 1 & 20 & & 2 & 23 & & & & 1 & 1 & & & & 1 & 1 \\
\hline Bostrichidae & & & & 7 & 7 & & & & 7 & 7 & & & & 11 & 11 \\
\hline Scolytidae & & & & 4 & 4 & & & & 4 & 4 & & & & 3 & 3 \\
\hline Carabidae & 4 & & & & 4 & & & & & 0 & 1 & & & & 1 \\
\hline Staphylinidae & 1 & & & & 1 & 4 & & & & 4 & & & & & 0 \\
\hline Scirtidae & & & & 1 & 1 & & & & 1 & 1 & & & & & 0 \\
\hline Passalidae & & & & 1 & 1 & & & & 1 & 1 & & & & & 0 \\
\hline Elateridae & 1 & & & & 1. & & & & 1 & 1 & & & & & 0 \\
\hline Tenebrionidae & 1 & & & & 1 & & & & & 0 & & & & & 0 \\
\hline $\begin{array}{l}\text { Não } \\
\text { Identificado }\end{array}$ & & & & & 0 & 2 & & & 2 & 4 & 1 & 2 & & 2 & 5 \\
\hline
\end{tabular}

Fonte - Moreira (2013). 
Estes besouros, de acordo com Koller et al. (1999) são importantes em estudos de fragmentos florestais, pois seu recurso alimentar é composto por frutas, fezes e carcaça dos vertebrados. Sendo também indicadores importantes de perturbações no ambiente onde vivem. Por isto, este grupo é indicado como componente essencial na manutenção dos ecossistemas.

Vital (2007) ressaltou que a monocultura não é capaz de fornecer a mesma amplitude de produtos e recursos existentes nas florestas nativas, o que pode justificar a baixa diversidade no cultivo de café. De acordo com Freitas \& Barreto (2008), os Coleópteros são altamente sensíveis a mudanças antrópicas, tendo efeito negativo principalmente pela agricultura.

De acordo com Medri \& Lopes (2001) as condições ambientais das florestas naturais diferem-se bruscamente daquelas presentes em uma área de cultivo. A alteração de ambientes naturais para pastagens ou monoculturas, ocasiona modificações nos fatores abióticos, o que, por sua vez, atinge fortemente as comunidades presentes, podendo consequentemente ocorrer declínio e ou eliminação de espécies que não se estabelecem nas novas condições do habitat. Freitas \& Barreto (2008) afirmam que a presença de indicadores biológicos em maior número em um local indica que este está sujeito a uma maior ciclagem e reposição de nutrientes.

Ao avaliar a biodiversidade do Fragmento florestal foi obtido o valor de $H^{\prime}=0,9680$ para Shannon (Figura 3). As condições favoráveis encontradas no Fragmento, tais como; a cobertura vegetal e a umidade geram um mosaico de condições micro climáticas que favorecem a permanência de diversos grupos, mantendo assim a biodiversidade local.

Moço (2005) relatou através de um de seus estudos que a cobertura vegetal de floresta natural, mostrou valores de densidade e riqueza de fauna, superiores aos de povoamento de monoculturas. Alves et al. (2008) afirmam que pode ter influência pela quantidade e qualidade da cobertura do solo que atuam ativamente na abundância e na diversidade dos organismos.

Em geral, ambas as monoculturas apresentaram uma menor riqueza comparado com o fragmento florestal. Isto pode estar associado aos microclimas gerados no fragmento, decorrente das eventuais precipitações da primavera, que além de estimular o crescimento vegetal, também favorecem a decomposição da matéria orgânica, aumentando assim a oferta de alimentos para a fauna, criando um ambiente 
favorável para o crescimento da biota do solo e estimulando a atividade biológica.

Em termos de riqueza geral, ao analisar somente as monoculturas, a Monocultura 1 apresentou 9 famílias seguida pela Monocultura 2 com 7 famílias. Apesar da monocultura 1 apresentar maior quantidade de famílias, a biodiversidade foi maior na segunda monocultura (H' 0,4238).

Este resultado foi possível devido principalmente a presença de famílias na monocultura $2 \mathrm{com}$ uma distribuição mais homogenia, sem a discrepância acentuada da família Scarabaeidae da Monocultura 1, H' 0,4209 (Figura 3). Ao calcular o índice de Pielou verificou-se que o fragmento apresenta maior equabilidade com o valor de $\mathrm{J}^{\prime}=0,1665$ e 0,0689 e 0,0714 para monocultura 1 e 2 respectivamente (Figura 3).

Figura 3. Índices de Shannon e Pielou das áreas amostradas, com respectivos intervalos de confiança a 95\% de probabilidade. Comunidade rural Mato Dentro, Campos Gerais, MG.

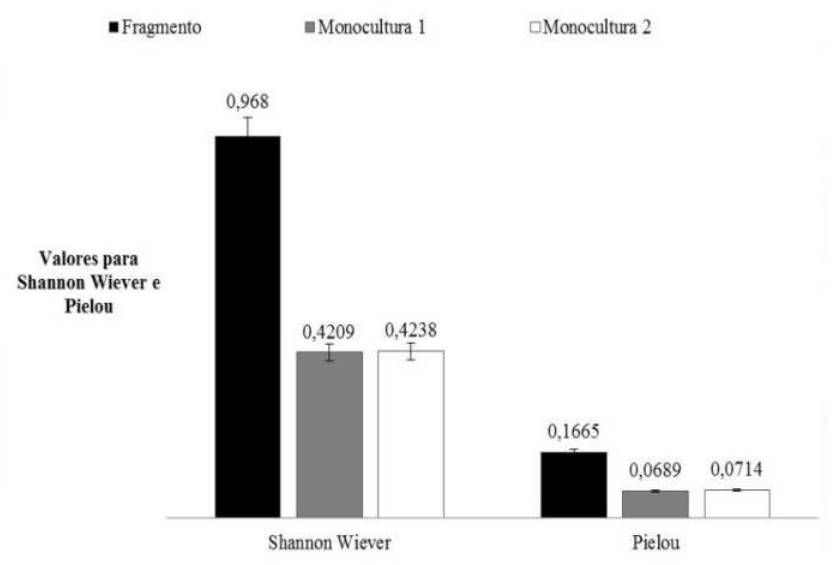

Fonte - Moreira (2013).
O fragmento apesar de também possuir grande representatividade da família Scarabaeidae, apresentou alto valor para o índice de Pielou, de acordo com Carbone (2010) isso pode estar relacionado a seu número menor de representantes desta família, o fragmento apresentou 256 indivíduos número menos representativo que as monoculturas 1 com 413 e a monocultura 2 com 345 escarabeídeos amostrados, ou seja, melhor distribuição neste local.

Nas 3 áreas estudadas, as iscas compostas por excrementos humanos foram mais atrativas aos besouros, tanto em termos de riqueza quanto abundância, sendo a família Scarabaeidae mais amostrada (Figura 4). Silva \& Vidal (2007) descrevem, que estes insetos são besouros de grande importância por atuarem como controladores de dípteros e nematoides gastrointestinais. Esse controle biológico é devido ao seu comportamento de retirar e enterrar excrementos de vertebrados no solo fazendo assim galerias na massa fecal e abaixo dela. Deste modo, estes insetos passam a competir com os parasitas pelo recurso. 
Figura 4. Número de famílias amostradas por pitfalls com diferentes atrativos. *PFH. Pitfall - Fezes Humanas; PFB - Pitfall - Fezes Bovinas; PC. PitfallControle. Comunidade rural Mato Dentro, Campos Gerais, MG.

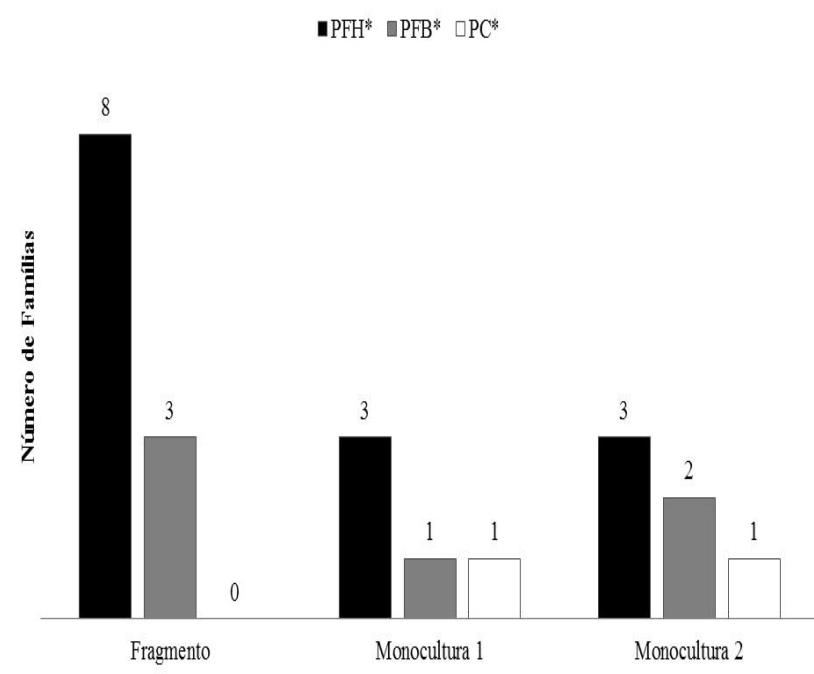

Fonte - Moreira (2013)

Estes besouros escarabeídeos presentaram-se com significativa preferência a fezes humanas, pois dos 1014 escarabeídeos coletados com pitfall, 914 foram amostrados nas armadilhas que continham fezes humanas. Essa diferença também permaneceu na soma total por área da coleta, o fragmento com 256 escarabeídeos, 252 destes foram atraídos por este atrativo. Nas monoculturas, esse resultado ocorreu da mesma forma, na monocultura 1 foram amostrados 413 escarabeídeos e destes 392 foram coletados com o uso de excrementos humanos. $\mathrm{Na}$ monocultura 2 o resultado continua contrastante, pois 270 besouros escarabeídeos foram coletados com iscas de fezes humanas, comparado aos 345 do total amostrado.

As iscas com fezes bovinas não apresentaram grande atratividade, pois no fragmento, na monocultura 1 e na monocultura 2 apresentaram 2, 16 e 70 escarabeídeos respectivamente. Já a pitfall controle que não apresentava nenhum atrativo, capturou apenas 7 escarabeídeos, o que corrobora a eficácia da utilização das iscas na captura dos besouros.

A atratividade das fezes humanas já foi observada Milhomen et al. (2003), segundo os autores, armadilhas de queda com isca de fezes humanas são as mais indicadas ao estudo dos escarabeídeos.

Filgueiras et al. (2009), ao analisarem a atratividade de Scarabaeidae por diferentes atrativos (fezes humanas, fezes de jaguar e antílope), no Parque Estadual de Dois Irmãos, um remanescente da Mata Atlântica Brasileira no estado de Recife município de Pernambuco, constataram que do total das amostragens, $80,5 \%$ dos escarabeídeos coletados foram amostrados com as iscas derivadas de fezes humanas. E mesmo quando oferecidos os dois atrativos, fezes humanas e bovinas, as fezes humanas são rapidamente atacadas por uma grande variedade de espécies de Scarabaeinae (HALFFTER \& 
MATTHEWS, 1966 apud FILGUEIRAS et al., 2009).

Essa preferência pelo atrativo pode estar relacionada ao habito alimentar do animal que forneceu os excrementos, em estudos de apud FILGUEIRAS et al., 2009 comparando iscas de animais carnívoros com iscas de herbívoros, observaram que as iscas derivadas de animais onívoros foram mais atraentes para os Coleópteros.

Ao comparar os dados da armadilha Pet Santa Maria, o Fragmento apresentou a maior quantidade de famílias amostradas, totalizando 8 famílias, seguido respectivamente pela monocultura $1 \operatorname{com} 6$ e a monocultura 2 com 5 famílias amostradas.

A família mais representativa neste método foi a Cerambycidae com 40 dos 87 indivíduos coletados. Ao calcular a diversidade pelo índice de Shannon notou-se uma maior diversidade no fragmento florestal com $\mathrm{H}^{\prime}=1,69$, seguido pela monocultura 1 com H' $=1,37$ e monocultura 2 apresentando $\mathrm{H}^{\prime}=1,15$. O que continua mostrando que o fragmento florestal oferece as melhores condições para a permanência dos besouros como dito por Vital (2007).

Ao comparar os dados da armadilha Pet Santa Maria, o Fragmento apresentou a maior quantidade de famílias amostradas, totalizando 8 famílias, seguido respectivamente pela monocultura 1 com 6 e a monocultura 2 com 5 famílias amostradas (Figura 5).

Figura 5. Distribuição das famílias amostradas por Pet Santa Maria nas 3 áreas de coleta. Comunidade rural Mato Dentro, Campos Gerais, MG.

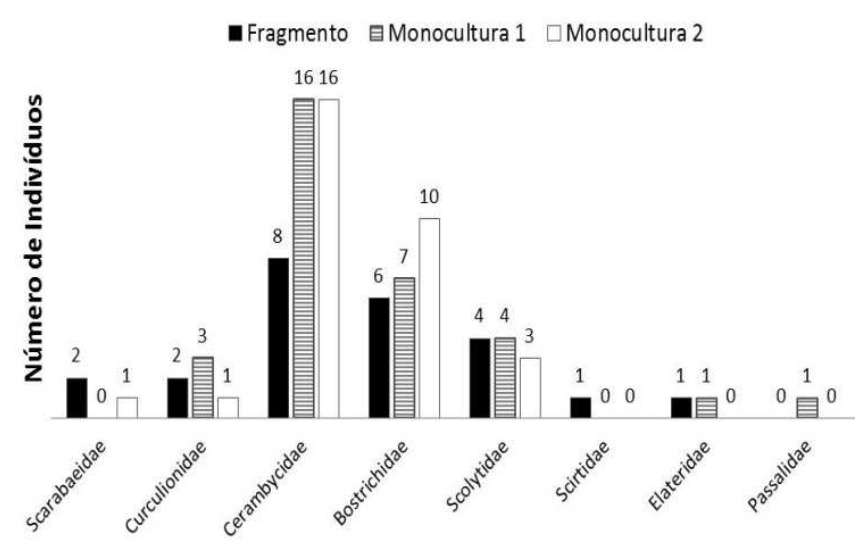

Fonte - Moreira (2013).

Ao calcular a diversidade pelo índice de Shannon notou-se uma maior diversidade no fragmento florestal com $\mathrm{H}^{\prime}=1,69$, seguido pela monocultura 1 com $\mathrm{H}^{\prime}=1,37 \mathrm{e}$ monocultura 2 apresentando $H^{\prime}=1,15$. Mantendo a afirmação que o fragmento florestal oferece as melhores condições para a permanência dos besouros, assim como dito por Vital (2007).

\section{CONCLUSÃO}

A maior diversidade de coleoptera foi encontrada no fragmento florestal, isso por possuir maior nível de preservação. A maior abundância foi observada nas monoculturas, o que relacionamos com a eventual presença do 
gado nos locais de coleta, fornecendo excrementos para a permanência dos besouros.

Essas considerações nos levaram a concluir que a alteração de um ambiente influi fortemente na composição dos organismos que habitam o local, o que foi visto na comparação do fragmento com as monoculturas. Ao comparar as monoculturas foi visto o oposto, pelo menos quanto à abundância, o fato de uma das monoculturas estar em processo de poda, essa alteração pode ter favorecido a coleta de besouros no local.

Houve grande diferença tanto para diversidade quanto para abundância de coleoptera quanto aos tipos de iscas utilizadas. A isca composta por excrementos humanos foi mais atrativa quando comparada com a de origem bovina, confirmando que iscas (fezes) derivadas de animais onívoros são mais atrativas a essa ordem de insetos.

\section{REFERÊNCIAS BIBLIOGRÁFICAS}

ALVES, M.V.; SANTOS, J. C. P.; GOIS, D. T.de; ALBERTON, J. V.; BARETTA, D. Macrofauna do Solo Influenciada Pelo Uso de Fertilizantes Químicos e Dejetos de Suínos no Oeste do Estado de Santa Catarina. Revista Brasileira de Ciência do Solo, 32:589-598, Viçosa - MG, 2008.
AQUINO, A. M ; AGUIAR-MENEZES, E. de A. \& QUEIROZ, J.M. Recomendações para coleta de artrópodes terrestres por armadilhas de queda ("Pitfall-Traps"), Soropédica, RJ, Dezembro 2006.

AUDINO, L., D, SILVA, P. G. DA, NOGUEIRA, J. M, MORAES, L. P. DE, \& VAZ-DE-MELLO, F. Z. Scarabaeinae (Coleoptera, Scarabaeidae) de um bosque de eucalipto introduzido em uma região originalmente campestre. Iheringia. Série Zoologia, 101(1-2), 121-126. 2011.

BIANCHIM, I.; ALVES, R.G.O.; KOLLEN, W.W.; Efeitos de Carrapaticidas/Inseticidas "Pour-On" sobre adultos do besouro coprófago Africano Onthophagus gazebela, Fabr: (Coleoptera: Serabaeidae). Sociedade Entomológica Brasileira. 27(2):275-279. 1998

CASTRO, C.R.T.; LEITE, H.G.; COUTO, L. Sistemas silvipastoris no Brasil: Potencialidades e entraves. Revista Árvore, v.20, n.4, p.575$582,1996$.

CARBONE, A. V. S. Levantamento Florístico e Fitossociológico de Matas Localizadas em Margens não Inundáveis do Rio Mogi Guaçu nos Municípios de Inconfidentes, Tocos do Moji e Bom Repouso-MG. Inconfidentes: IF do SUL de MINAS, 2010. 26 p. MonografiaGraduação Tecnológica em Gestão Ambiental, Instituto Federal de Educação, Ciência e Tecnologia do Sul de Minas Gerais - Campus Inconfidentes, Inconfidentes, 2010.

COPATTI, C. E.; DAUT, C. R. Diversidade de artrópodes na serapilheira em fragmentos de mata nativa e Pinus elliottii (Engelm. Var elliottii). Ciência e Natura, UFSM, 31 (1): 95 $113,2009$. 
COSTA, C.M.Q., SILVA, F.A.B., FARIAS, A.I.; MOURA, R.C. Diversidade de Scarabaeinae (Coleoptera, Scarabaeidae) coletados com armadilha de interceptação de vôo no refúgio ecológico Charles Darwin, Igarassu-PE, Brasil. Revista Brasileira de Entomologia.53(1):88-94. 2009.

DAVIS, A. L. V. Diversidade de escaravelhos na África do Sul: fatores influentes ao estado de conservação e inadequações de dados a pesquisa. Entomologia Africana 10: 53-65. 2002.

DOUBE, B.M.; WARDHALGH, K.G. Associações de habitats e nichos ecológico da comunidade de besouros coprófagos. Acta Oecologica., v.12, p.451-459, 1991.

FILGUEIRAS, B. K. C.; LIBERAL, C. N.; AGUIAR, C. D. M.; HERNÁNDEZ, M. I. M.; IANNUZZI, L. Attractivity of omnivore, carnivore and herbivore mammaliandung to Scarabaeinae (Coleoptera, Scarabaeidae) in a tropical Atlantic rainforest remnant. Revista Brasileira de Entomologia 53(3):422-427, setembro 2009.

FREITAS, A. C. S.; BARRETO, L. V. Qualidade Biológica do Solo em Ecossistemas de Mata Nativa e Monocultura do Café. Instituto Construir e Conhecer. Goiânia - GO. Enciclopédia Biosfera N.05, 2008.

KOLLER, W. W.; GOMES, A.; RODRIGUES, S. R. ; ALVES, R. G. de O. Besouros Coprófagos (Coleoptera: Scarabaeidae) Coletados em Campo Grande, MS, Brasil. Anais Sociedade de Entomologia do Brasil 28(3). Setembro, 1999.
MEDRI, I.M.; LOPES, J. Scarabaeidae (Coleoptera) do Parque Estadual Mata dos Godoy e de área de pastagem, no norte do Paraná, Brasil. Revista brasileira de Zoologia, 18(Supl.1): 135-141. 2001.

MILHOMEM, M.S.; VAZ-DE-MELLO, F. Z.; DINIZ,I.R. Técnicas de coleta de besouros copronecrófagos no Cerrado. Pesquisa agropecuária brasileira, v.38, n.11, p.12491256, 2003.

MOÇO, M. K. Caracterização da Fauna Edáfica em Diferentes Coberturas Vegetais na Região Norte Fluminense. Revista Basileira de Ciência do Solo, vol.29 no.4. Viçosa - MG, 2005.

NAZARO, C. M. N.; MORIYA M. R.; ALBUQUERQUE, L. B. Macrofauna edáfica bioindicadora de qualidade de solo em sistemas orgânicos de produção de café sombreado e a pleno sol. Anais do encontro de iniciação científica - ENIC. Universidade Estadual de Mato Grosso do Sul - UEMS. n. 2 (2010).

OLIVEIRA, G.. Uma descrição agroecológica da crise atual. Revista Nera, América do Norte, Ano 12, No. 15 pp 66-87. 2012.

OLIVEIRA, C. M.; MENDONÇA, J. S. F. Técnicas de Coleta de Scarabaeoidea (Insecta: Coleoptera): dispositivo antipilhagem de iscas em armadilhas de queda. Comunicado Técnico ISSN online 2176-5073 Planaltina, DF Março, 2011

VITAL, M. H. F. Impacto Ambiental de Florestas de Eucalipto. Revista do BNDES, Rio de Janeiro, V. 14, N. 28, P. 235-276, dez. 2007. 